\title{
Induced Charge Electrophoresis of a Conducting Cylinder in a Cylindrical Chamber
}

\author{
Huicheng Feng, Teck Neng Wong ${ }^{*}$, Marcos \\ School of Mechanical and Aerospace Engineering, Nanyang Technological University, \\ 50 Nanyang Avenue, Singapore 639798 \\ *Corresponding Author: mtnwong @ntu.edu.sg
}

\begin{abstract}
Induced charge electrophoresis (ICEP) of a conducting cylinder suspended in a cylindrical chamber is analytically studied in this paper. The results show that microvortices are generated around the cylinder, which is useful for fluid mixing and/or regulating in micro/nanofluidics. Furthermore, when the cylinder is off the center of the cylindrical chamber, it is driven into motion by the dielectrophoretic (DEP) force due to the non-uniform electric field and the ICEP force due to the asymmetric fluid flow. We discover that the cylinder not only translates but also rotates. By prohibiting the cylinder from translation but not rotation, a micro-motor could be generated based on the ICEP rotation of the cylinder. The velocities increase as the eccentricity increases and obtain peak values with the variation of the radius ratio.
\end{abstract}

Keywords: induced charge electrophoresis, microvortex generation, micro-motor.

\section{Introduction}

Electrokinetic phenomena occurred on the polarizable (conducting/dielectric) particle/surface are due to the interactions between the polarization charges and the applied electric field ${ }^{(1)}$. When the surrounding electric field is non-uniform, the dipoles in the polarizable particles experience an electric force, which drives the particle into motion. This kind of particle motion is referred to as dielectrophoresis (DEP). The ions in the electrolyte solution are attracted to the polarized surface, forming an induced electric double layer (EDL). The interactions between the induced EDL and the applied electric field produce a quadratic fluid flow referred to as Induced charge electroosmosis $(\mathrm{ICEO})^{(2)}$. When the ICEO flow is asymmetric, the particle moves via induced charge electrophoresis (ICEP) ${ }^{(3)}$.

Induced charge electrokineitc (ICEK) phenomena, including ICEO and ICEP, have been widely studied due to the promising potential applications in micro/nanofluides ${ }^{(3)}$, such as mixing ${ }^{(4-6)}$, demixing ${ }^{(7)}$, pumping $^{(8,9)}$, particle manipulation $^{(10,11)}$ and motion generation ${ }^{(10)}$. ICEP motion of the conducting particles has attracted lots of attentions. Particle-particle interactions ${ }^{(12-15)}$ and particle-wall interactions $^{(15-18)}$ have been analyzed under DC/AC electric field for spherical/cylindrical particles. In this paper, the motion of a conducting cylinder suspended in a cylindrical chamber due to ICEP and DEP is studied under bipolar coordinate system. The ICEO flow around the cylinder and the velocities of the cylinder are presented.

\section{Mathematical Model}

\subsection{Problem statement}

We hereby consider an infinite conducting cylinder suspended in an insulating cylindrical chamber filled by electrolyte solution. The EDL adjacent to the cylinder's surface is assumed to be much thinner than the radius of the cylinder and the distance between the cylinder and cylindrical chamber. We adopt bipolar coordinate system $(\tau, \sigma)$ to conveniently describe the complex geometry (Fig. 1). The relationship between the Cartesian coordinate and bipolar coordinate systems is given by ${ }^{(19)}$,

$$
x=a \frac{\sinh \tau}{\cosh \tau-\cos \sigma}, y=a \frac{\sin \sigma}{\cosh \tau-\cos \sigma}
$$

where $-\infty<\tau<\infty, 0<\sigma \leq 2 \pi ; a$ is a positive constant in bipolar coordinates. We use $\tau=\tau_{i}$ and $\tau=\tau_{o}$ to represent the surfaces of cylinder and cylindrical chamber, respectively (Fig. 1). 


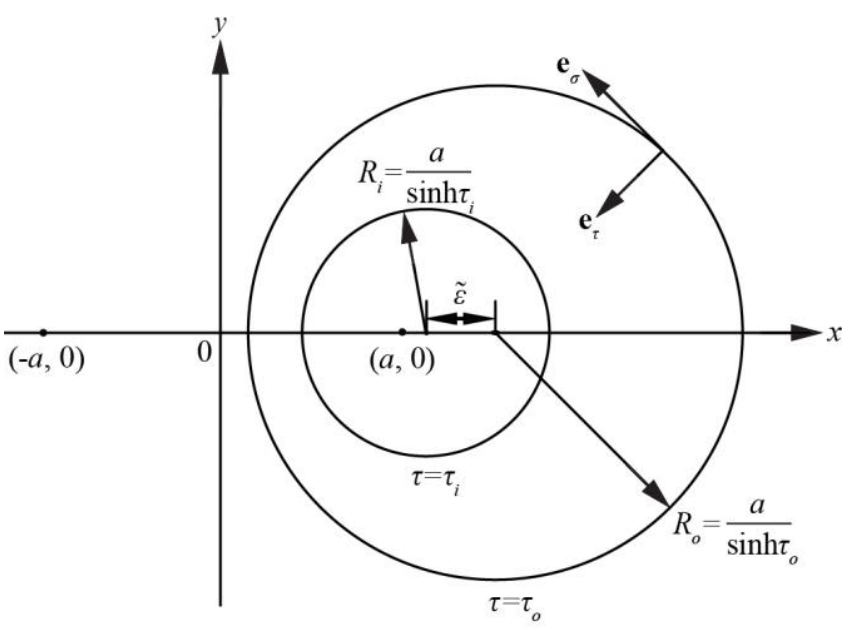

Fig. 1 Schematic diagram of the cylinder suspended in a cylindrical chamber.

\subsection{Governing equations}

Subjecting the cylindrical chamber to an electrical potential $E_{0} R_{o} \sin \left(\sigma+\sigma_{0}\right)$, where $E_{0} R_{i}<k_{B} T /(z e)$, the cylinder is polarized immediately after the establishment of the electric field. The ions are attracted to the cylinder surface and an EDL is formed within a very short time $\left(\sim 10^{-4} \mathrm{~s}\right)^{(2)}$. The bulk solution outside the EDL remains electrically neutral. Therefore, the electrical potential in the bulk solution is governed by the Laplace equation,

$$
\nabla^{2} \emptyset=0
$$

No electric field lines enter the EDL, thus no-flux condition is applied on the cylinder,

$$
\mathbf{e}_{\tau} \cdot \nabla \varnothing=0 \text { at } \tau=\tau_{i}
$$

The induced zeta potential $\zeta$ on the cylinder's surface depends on the external electric field by the following equation $^{(20)}$,

$$
\zeta=-\emptyset_{e}+\emptyset_{c}
$$

where $\emptyset_{e}$ is the external electrical potential, $\emptyset_{c}$ is an integral constant defined as $\emptyset_{c}=\int_{S} \emptyset_{e} d A / A$, where $A$ is the entire surface of the cylinder.

The DEP force and moment per unit length exerted on the cylinder are ${ }^{(21)}$,

$$
\begin{gathered}
\boldsymbol{F}_{D E P}=\int_{A}\left(\Pi_{\boldsymbol{e}} \cdot \mathbf{e}_{\boldsymbol{n}}\right) d A \\
\boldsymbol{M}_{D E P}=R \int_{A} \mathbf{e}_{\boldsymbol{n}} \times\left(\Pi_{\boldsymbol{e}} \cdot \mathbf{e}_{\boldsymbol{n}}\right) d A
\end{gathered}
$$

where $\mathbf{e}_{n}$ is the unit vector normal to the cylinder surface; $\Pi_{\boldsymbol{e}}$ is the Maxwell stress tensor,

$$
\Pi_{\boldsymbol{e}}=\varepsilon_{w}\left(\boldsymbol{E} \boldsymbol{E}-\frac{1}{2} E^{2} \mathbf{I}\right)
$$

Reynolds number is very small in ICEO flow ${ }^{(2)}$ and the fluid is incompressible. Thus the Stokes equations are applicable. The general solution of the stream function $\varphi$ in bipolar coordinates suitable for the present case was given by Jeffery ${ }^{(22)}$,

$$
\begin{aligned}
h \varphi & =A_{0} \cosh \tau+B_{0} \tau(\cosh \tau-\cos \sigma) \\
& +C_{0} \sinh \tau+D_{0} \tau \sinh \tau+h_{1} \tau \sin \sigma \\
& h \sum_{n=1}^{\infty}\left\{a_{n} \cosh (n+1) \tau+b_{n} \cosh (n-1) \tau\right. \\
& \left.c_{n} \sinh (n+1) \tau+d_{n} \sinh (n-1) \tau\right\} \cos n \sigma \\
& +h \sum_{n=1}^{\infty}\left\{e_{n} \cosh (n+1) \tau+f_{n} \cosh (n-1) \tau\right. \\
& +h \sum_{n=1}^{\infty}\left\{e_{n} \cosh (n+1) \tau+f_{n} \cosh (n-1) \tau\right. \\
& \left.g_{n} \sinh (n+1) \tau+h_{n} \sinh (n-1) \tau\right\} \sin n \sigma
\end{aligned}
$$

where $h=(\cosh \tau-\cos \sigma) / a$.

The established electric field exerts an electrical force on the ions within the EDL, which drives the ions as well as the bulk fluid into motion. The slip velocity on the cylinder surface is defined by the Helmholtz-Smoluchowski formula,

$$
\mathbf{u}_{s}=\frac{\varepsilon_{w} \zeta}{\mu} E_{\|} \mathbf{e}_{t} \text { at } \tau=\tau_{i}
$$

where the zeta potential $\zeta$ and the electric field on and tangent to the cylinder surface $E_{\|}$are determined by the established electric field.

No-slip boundary condition is applied on the cylindrical chamber,

$$
\mathbf{u}=0 \text { at } \tau=\tau_{o}
$$

The ICEP force and moment due to the surrounding ICEO flow can be obtained from Eq. (5) and Eq. (6) by replacing Maxwell stress tensor [Eq. (7)] with the viscous stress tensor, $\Pi_{H}=-p \mathbf{I}+\mu\left[\nabla \boldsymbol{u}+(\nabla \boldsymbol{u})^{\boldsymbol{T}}\right]$. The drag force and moment due to the cylinder motion with velocity $U_{x} \mathbf{e}_{x}+U_{y} \mathbf{e}_{y}+\Omega R_{i} \mathbf{e}_{\sigma}$ can also be obtained through Eq. (5) and Eq. (6). The cylinder is in free suspension, thus the net force and moment exerted on it should vanish,

$$
\boldsymbol{F}_{D E P}+\boldsymbol{F}_{I C E P}+\boldsymbol{F}_{d}=0, \boldsymbol{M}_{D E P}+\boldsymbol{M}_{I C E P}+\boldsymbol{M}_{d}=0
$$

Solving these equations together, the electric and fluid flow fields and the cylinder velocities are determined,

$$
\begin{aligned}
U_{x} & =\frac{1}{8} U_{i} \sinh \tau_{i}\left[\left(\tau_{i}-\tau_{o}\right)-\tanh \left(\tau_{i}-\tau_{o}\right)\right. \\
& \left.+2 \tanh \left(\tau_{i}-\tau_{o}\right) \cos 2 \sigma_{0}\right] /\left[\sinh ^{2} \tau_{o}\right.
\end{aligned}
$$




$$
\begin{aligned}
& \left.\times \cosh ^{2}\left(\tau_{i}-\tau_{o}\right)\right] \\
U_{y}= & -\frac{1}{8} U_{i} \cosh \tau_{i} \tanh \left(\tau_{i}-\tau_{o}\right) \sin 2 \sigma_{0} /\left[\sinh ^{2} \tau_{o}\right. \\
\times & \left.\cosh ^{2}\left(\tau_{i}-\tau_{o}\right)\right] \\
\Omega= & -\frac{1}{8} \frac{U_{i}}{R_{i}} \tanh \left(\tau_{i}-\tau_{o}\right) \sin 2 \sigma_{0} /\left[\sinh ^{2} \tau_{o}\right. \\
& \left.\times \cosh ^{2}\left(\tau_{i}-\tau_{o}\right)\right]
\end{aligned}
$$

The cylinder velocities are Trigonometric functions of the electric field phase angle $\sigma_{0}$. Thus the cylinder velocities (both of the direction and magnitude) can be manipulated by changing $\sigma_{0}$.

\section{Results and Discussion}

\subsection{ICEO flow field}

To facilitate the following analysis, we hereby introduce two parameters, radius ratio $R_{r}$ and eccentricity $\varepsilon$,

$$
R_{r}=\frac{R_{i}}{R_{o}}, \quad \varepsilon=\frac{\tilde{\varepsilon}}{R_{o}-R_{i}}
$$

Fig. 2 depicts the flow patterns around the stationary cylinder with $\sigma_{0}=0, R_{r}=0.1, \varepsilon=0.5$. The flow contains four counter-rotating vortices. Due to the eccentricity, the ICEO flow is no longer symmetric to the $y$-axis. The two vortices on the left hand side are smaller than the two on the right hand side. The asymmetric ICEO flow exerts hydrodynamic force and/or moment on the cylinder. When it is free to go, it would translate and/or rotate. Once the cylinder reaches the center of the cylindrical chamber (the cylinder and cylindrical chamber become concentric), the ICEO flow regains perfect symmetry. The cylinder experiences no force/moment and becomes stationary. The detailed studies on the concentric case can be referenced from Zhao and $\mathrm{Bau}^{(6)}$. The velocity scale $U_{i}$ used in Fig. 2 is $U_{i}=\varepsilon_{w} E_{0}^{2} R_{i} / \mu$.

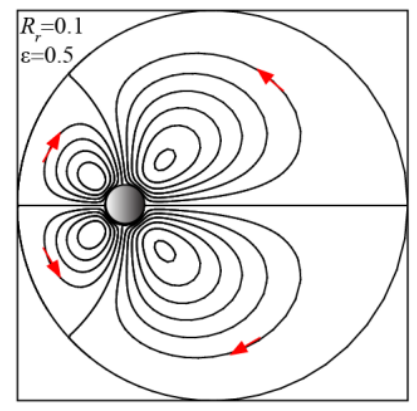

Fig. 2 ICEO flow (streamlines) around the stationary conducting cylinder. The increment between the neighboring streamlines is $\varphi /\left(U_{i} R_{i}\right)=0.2$. Here $\sigma_{0}=0$.

\subsection{Cylinder velocities}

Fig. 3 presents the variations of cylinder velocities with the radius ratio $R_{r}$ at different eccentricities $\varepsilon$ with electric field phase angle $\sigma_{0}=\pi / 6$. Under this condition, $U_{x}$ is positive; $U_{y}$ and $\Omega$ are negative. The directions of these velocities as well as the magnitudes can be manipulated by changing $\sigma_{0}$. Fig. 3 clearly presents that all the cylinder velocities diminish to zero at both $R_{r}=0$ and $R_{r}=1.0$, and the magnitudes of the velocities monotonically increase as $\varepsilon$ increases. The cylindrical chamber becomes very large when $R_{r}$ approaches 0 , so as the distance between the cylinder and cylindrical chamber. Hence the influence of the cylindrical chamber on the cylinder motion becomes negligible. Once $R_{r}$ reaches 0 $\left(\tau_{o}=0\right)$, the cylindrical chamber becomes a planar plate wall. For detailed studies on this situation, please reference to Zhao and $\mathrm{Bau}^{(6)}$. The annulus space becomes extremely small when $R_{r}$ approaches 1 , hence the viscous effect increases tremendously. Thus the cylinder motion decreases or even vanishes. However, one should notice that the EDLs on the cylinder and cylindrical chamber might become interacting with each other when $R_{r}$ approaches 1, which invalidates the thin EDL approximation. Such extreme situation is beyond the scope of the present model.
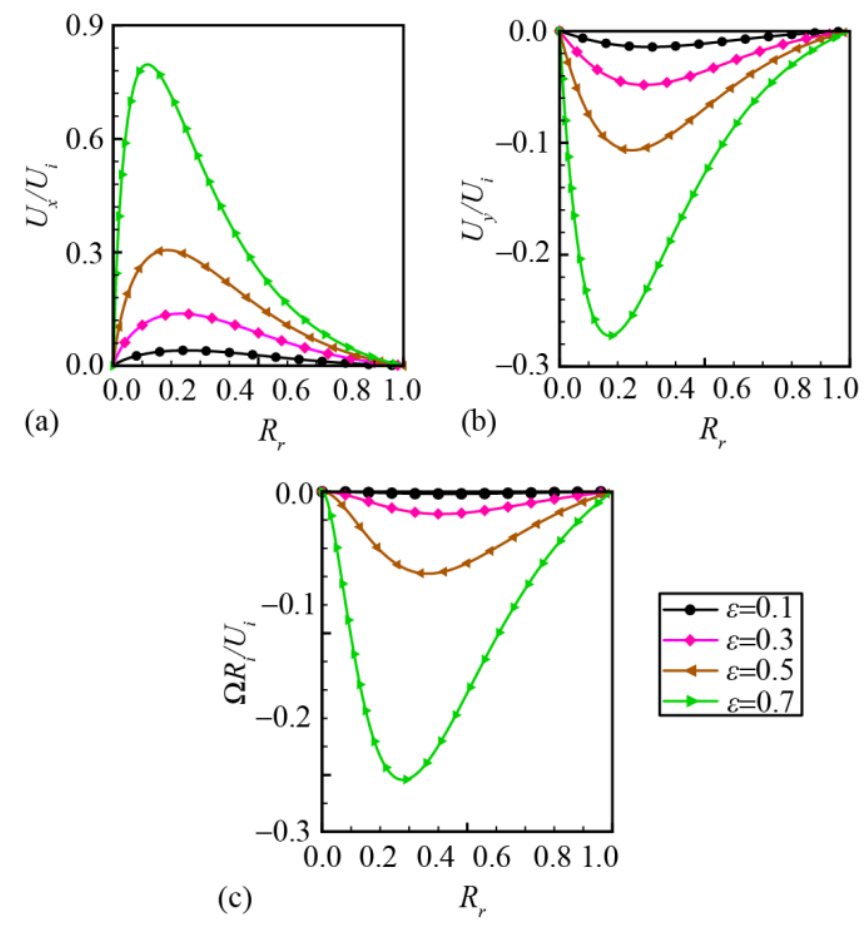

Fig. 3 Variations of cylinder velocities with the radius ratio $R_{r} \quad$ at different eccentricities $\varepsilon$. Here $\sigma_{0}=\pi / 6$. 
Table 1. Typical parameters of the ICEP system for $10^{-5} \mathrm{M}$ KCl solution.

\begin{tabular}{|c|c|c|}
\hline Dielectric constant & $\varepsilon_{w}$ & $7 \times 10^{-7} \mathrm{~g} \mathrm{~m} \mathrm{~V}^{-2} \mathrm{~s}^{-2}$ \\
\hline Viscosity & $\mu$ & $1 \mathrm{~g} \mathrm{~m}^{-1} \mathrm{~s}^{-1}$ \\
\hline Ion diffusivity & $D_{i}$ & $10^{-9} \mathrm{~m}^{2} \mathrm{~s}^{-1}$ \\
\hline Debye length & $\lambda_{D}$ & $100 \mathrm{~nm}$ \\
\hline Cylinder radius & $R_{i}$ & $10 \mu \mathrm{m}$ \\
\hline Charging time & $t_{c}=\lambda_{D} R_{i} / D_{i}$ & $10^{-3} \mathrm{~s}$ \\
\hline
\end{tabular}

\subsection{ICEP micro-motor}

As shown in Fig. 3 (c), the cylinder rotates when it does not share the same center with the cylindrical chamber. Utilizing the typical parameters in Table 1 and applying an electric field of strength $E_{0}=10^{3} \mathrm{Vm}^{-1}$, the translational velocity scale of the cylinder is small, $U_{i}=7 \times 10^{-6} \mathrm{~m} \mathrm{~s}^{-1}$. The motion time scale is $t_{0}=$ $R_{i} / U_{i}=1.4 \mathrm{~s}$, which is large compared to the charging time $t_{c}=10^{-3} \mathrm{~s}$ (Table 1 ). Thus we can assume that the translation and rotation of the cylinder do not affect the EDL establishment on the cylinder.

Prohibiting the cylinder from translation but not rotation, a micro-motor could be generated due to the ICEP rotation. The rotational velocity scale is $\Omega_{i}=\varepsilon_{w} E_{0}^{2} / \mu$, which is free of the cylinder radius. The rotational velocity of the cylinder $\Omega_{i}$ is a quadratic function of the electric field strength $E_{0}$ (Fig. 4). At $E_{0}=8.45 \times 10^{3} \mathrm{Vm}^{-1}$, the rotational velocity scale $\Omega_{i}$ is $50 \mathrm{rs}^{-1}$. The actual rotational velocity can be determined by choosing appropriate eccentricity $\varepsilon$ and radius ratio $R_{r}$ according to Fig. 3 (c). The larger the electric field strength $E_{0}$, the greater the rotational velocity scale $\Omega_{i}$. However, the weak applied electric field approximation $\left[E_{0} R_{i}<k_{B} T /(z e)\right]$ should be satisfied.

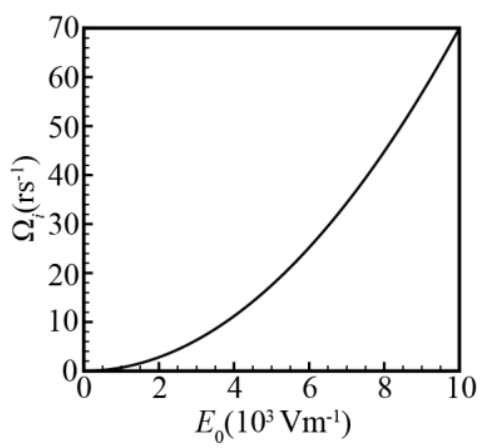

Fig. 4 Variation of rotational velocity scale $\Omega_{i}$ with respect to the electric field strength $E_{0}$.

\section{Conclusions}

The present analytical model characterizes the cylinder motion due to ICEP and DEP. The results reveal that microvortices are generated around the cylinder, which is useful for fluid mixing and/or regulating in micro/nanofluidics. Furthermore, we find the cylinder not only translates but also rotates. Micro-motor could be produced by letting the cylinder rotate but not translate. Larger eccentricity provides greater velocities. As radius ratio increases, the velocities obtain peak values.

\section{Acknowledgment}

The authors gratefully acknowledge research support from the Singapore Ministry of Education Academic Research Fund Tier 2 research Grant MOE2011-T2-1-036.

\section{References}

(1) Antonio Ramos, Electrokinetics and electrohydrodynamics in microsystems. Springer Wien, New York, 2011, pp. 62-63, 222-225

(2) Todd M. Squires, and Martin Z. Bazant: "Induced-charge electro-osmosis", J. Fluid Mech., Vol. 509, pp. 217-252, 2004

(3) Todd M. Squires, and Martin Z. Bazant: "Induced-charge electrokinetic phenomena", Curr. Opin. Colloid In., Vol. 15, No. 3, pp. 203-213, 2010

(4) Cindy K. Harnett, Jeremy. Templeton, Katherine. A. Dunphy-Guzman, Yehya M. Senousy, and Michael P. Kanouff: "Model based design of a microfluidic mixer driven by induced charge electroosmosis", Lab chip, Vol. 8, No. 4, pp. 565-572, 2008

(5) Zhemin Wu, and Dongqing Li: "Micromixing using induced-charge electrokinetic flow", Electrochim. Acta, Vol. 53, No. 19, pp. 5827-5835, 2008

(6) Hui Zhao, and Haim H. Bau: "Microfluidic chaotic stirrer utilizing induced-charge electro-osmosis", Phys. Rev. E, Vol. 75, No. 5, pp. 006217, 2007

(7) Felix C. Leinweber, Jan C.T. Eijkel, Johan G. Bomer, and Albert van den Berg: "Continuous flow microfluidic demixing of electrolytes by induced charge electrokinetics in structured electrode arrays", Anal. Chem., Vol. 78, No. 5, pp. 1425-1434, 2006

(8) Martin Z. Bazant, and Todd M. Squires: "Induced-charge electrokinetic phenomena: theory and microfluidic applications", Phys. Rev. Lett., Vol. 92, pp. 
066101, 2004

(9) Misha M. Gregersen, Fridolin Okkels, Martin. Z. Bazant, and Henrik Bruus: "Topology and shape optimization of induced-charge electro-osmotic micropumps", New J. Phys., Vol. 11, No. 7, pp. 075019, 2009

(10) Todd M. Squires, and Martin Z. Bazant: "Breaking symmetries in induced-charge electro-osmosis and electrophoresis", J. Fluid Mech., Vol. 560, pp. 65-101, 2006

(11) Sumit Gangwal, Olivier J. Cayre, Martin Z. Bazant, and Orlin D. Velev: "Induced-charge electrophoresis of metallodielectric particles", Phys. Rev. Lett., Vol. 100, pp. 058302, 2008

(12) David Saintillan, Eric Darve, and Eric S. G. Shaqfeh: "Hydrodynamic interactions in the induced-charge electrophoresis of colloidal rod dispersions", J. Fluid Mech., Vol. 563, pp. 223, 2006

(13) David Saintillan, Eric Darve, and Eric S.G. Shaqfeh: "Stabilization of a suspension of sedimenting rods by induced-charge electrophoresis", Phys. Fluids, Vol. 18, No. 12, pp. 121701, 2006

(14) Zhemin $\mathrm{Wu}$, and Dongqing Li: "Induced-charge electrophoretic motion of ideally polarizable particles", Electrochim. Acta, Vol. 54, No. 15, pp. 3960-3967, 2009

(15) Hideyuki Sugioka: "Basic analysis of induced-charge electrophoresis using the boundary element method", Colloid Surface A, Vol. 376, pp. 102-110, 2011

(16) Hui Zhao, and Haim H. Bau: "On the effect of induced electro-osmosis on a cylindrical particle next to a surface", Langmuir, Vol. 23, pp. 4053-4063, 2007

(17) Zhemin $\mathrm{Wu}$, Yandong Gao, and Dongqing Li: "Electrophoretic motion of ideally polarizable particles in a microchannel", Electrophoresis, Vol. 30, No. 5, pp. 773-781, 2009

(18) Martin Z. Bazant, and Mustafa Sabri Kilic: "Induced-charge electrophoresis near a wall", Electrophoresis, Vol. 32, No. 5, pp. 614-628, 2011

(19) Huan J. Keh, Kuo D. Horng, and Jimmy Kuo: "Boundary effects on electrophoresis of colloidal cylinders", J . Fluid Mech., Vol. 231, pp. 211-228, 1991

(20) Zhemin $\mathrm{Wu}$, and Dongqing Li: "Mixing and flow regulating by induced-charge electrokinetic flow in a microchannel with a pair of conducting triangle hurdles", Microfluid Nanofluid, Vol. 5, No. 1, pp. 65-76, 2007

(21) David Saintillan: "Nonlinear interactions in electrophoresis of ideally polarizable particles", Phys. Fluids, Vol. 20, No. 6, pp. 067104, 2008

(22) G. B. Jeffery: "The rotation of two circular cylinders in a viscous fluid", Proc. R. Soc. Lond. A, Vol. 101, No. 709, pp. 169-174, 1922 